\title{
Degenerescência da polpa e respiração de quivi cv. "Bruno" em função das condições de armazenamento
}

\author{
Internal breakdown and respiration of 'Bruno' kiwifruit in relation to storage conditions
}

\author{
Cristiano André Steffens ${ }^{I}$ Auri Brackmann"II Sidinei José Lopes ${ }^{\text {II }}$ \\ Josuel Alfredo Vilela Pinto ${ }^{\text {II }}$ Ana Cristina Eisermann ${ }^{\text {II }}$ \\ Ricardo Fabiano Hettwer Giehl ${ }^{\mathrm{II}}$ Anderson Webber ${ }^{\mathrm{II}}$
}

\section{RESUMO}

\begin{abstract}
O objetivo deste trabalho foi avaliar o efeito da atmosfera controlada sobre a ocorrência da degenerescência da polpa, de sabor e aroma alcoólico no quivi cultivar "Bruno" e a relação destes parâmetros com a taxa respiratória e o quociente respiratório. Os tratamentos foram 0,5; 1,0 e 1,5kPa $\mathrm{O}_{2}$ combinados com 8, 12 e $16 \mathrm{kPa} \mathrm{CO}_{2}$. A incidência de degenerescência da polpa e de frutos com aroma alcoólico foi menor nos frutos armazenados com $8 \mathrm{kPa}$ de $\mathrm{CO}_{2}$, independente do nível de $\mathrm{O}_{2}$. A taxa respiratória dos frutos foi menor nos tratamentos com $16 \mathrm{kPa}$ de $\mathrm{CO}_{2}$ e o quociente respiratório apresentou os maiores valores no tratamento com $0,5 \mathrm{kPa}$ de $\mathrm{O}_{2}$ combinado com $16 \mathrm{kPa}$ de $\mathrm{CO}_{2}$. A taxa respiratória correlacionou-se negativamente com a incidência de degenerescência da polpa e com a presença de aroma alcoólico. $O$ quociente respiratório apresentou uma correlação positiva com a degenerescência da polpa e com a incidência de aroma alcoólico. Segundo a análise sensorial, os tratamentos com 1,0 e $1,5 \mathrm{kPa}$ de $\mathrm{O}_{2}$ combinados com $8 \mathrm{kPa}$ de $\mathrm{CO}_{2}$, não induziram a formaçãò de sabor alcoólico nos frutos.
\end{abstract}

Palavras-chave: Actinidia chinensis, pós-colheita, distúrbios fisiológicos, atmosfera controlada.

\section{ABSTRACT}

This research was aimed at evaluating the effect of controlled atmosphere on the internal breakdown, alcoholic taste and flavor in 'Bruno' kiwifruits and the relationship between these parameters and the rate and respiratory quotient. The treatments were 0.5, 1.0 and $1.5 \mathrm{kPa} \mathrm{O}_{2}$ combined with 8 , 12 and $16 \mathrm{kPa} \mathrm{CO}$. The internal breakdown and occurrence of fruits with flavor was lower at $8 \mathrm{kPa}$ of $\mathrm{CO}_{2}$ independent of $\mathrm{O}$ level. Respiration rate of fruits was lower at treatments with $16 \mathrm{kPa}$ of $\mathrm{CO}_{2}$ and the respiratory quotient showed highest values at treatment with $16 \mathrm{kPa}$ of $\mathrm{CO}_{2}$. The respiration rate was negatively correlated with internal breakdown and flavor incidence. The respiratory quotient showed a positive correlation with internal breakdown and alcoholic aroma incidence. According to sensory analysis, the treatments with 1.0 and $1.5 \mathrm{kPa}$ of $\mathrm{O}_{2}$ combined with $8 \mathrm{kPa}$ of $\mathrm{CO}_{2}$ did not induce the of flavor taste in fruits.

Key words: Actinidia chinensis, postharvest, physiological disorders, controlled atmosphere.

\section{INTRODUÇÃO}

A durabilidade de um produto perecível na pós-colheita é dependente da sua sensibilidade à ação de microrganismos patogênicos, da taxa de transpiração e da sua atividade metabólica. Os principais fatores que influenciam a manutenção da qualidade dos frutos durante o armazenamento e, conseqüentemente, a redução das perdas pós-colheita são a redução da temperatura, a diminuição da pressão parcial de $\mathrm{O}_{2}$ e o aumento da pressão parcial de $\mathrm{CO}_{2}$ através da atmosfera controlada ou modificada. Essè efeito é devido à redução nos processos metabólicos, principalmente a respiração celular, que culmina no amadurecimento dos frutos (SHIINA et al., 1997).

O processo respiratório envolve uma série de reações de oxirredução, em que compostos orgânicos são oxidados a $\mathrm{CO}_{2}$, sendo este considerado o principal fator que contribui para as perdas póscolheita de produtos perecíveis (MAHAJAN \& GOSWANI, 2001). De acordo com esses autores, a diminuição da atividade enzimática pela baixa temperatura, pelo baixo $\mathrm{O}_{2}$ e/ou pelo alto $\mathrm{CO}_{2}$, em geral, reduz a utilização de substratos e aumenta a vida pós-

IDepartamento de Fitotecnia, Universidade do Estado de Santa Catarina (UDESC). Av. Luiz de Camões, 2090, Bairro Conta Dinheiro, 88520-000, Lages, SC, Brasil. E-mail: steffens@cav.udesc.br. Autor para correspondência.

IIDepartamento de Fitotecnia, Universidade Federal de Santa Maria (UFSM), Santa Maria, RS, Brasil. 
colheita dos frutos. No entanto, SAQUET \& STREIF (2000) afirmam que nem sempre frutos com baixa taxa respiratória, durante o armazenamento, apresentam um maior tempo de conservação. Isso ocorre devido ao fato de que o uso de pressões parciais de $\mathrm{O}_{2}$ excessivamente baixas ou altas de $\mathrm{CO}_{2}$ podem induzir a respiração anaeróbica (SHIINA et al., 1997), levando à formação e ao acúmulo de etanol e acetaldeído nos tecidos (KE et al., 1993), os quais são compostos tóxicos que podem desencadear o surgimento de degenerescência da polpa dos frutos e o desenvolvimento de aroma e sabor alcoólicos (SAQUET et al., 2000). Este problema torna-se ainda mais grave no armazenamento em atmosfera modificada, onde normalmente o $\mathrm{O}_{2}$ pode atingir níveis muito baixos $(<2 \mathrm{kPa})$ e o $\mathrm{CO}_{2}$ muito altos $(>10 \mathrm{kPa})$.

O sucesso da atmosfera modificada em manter a qualidade do produto depende da redução da respiração aeróbica, sem a ocorrência da indução da respiração anaeróbica (PETRACEK et al., 2002). Assim, para manter a qualidade dos frutos, durante o armazenamento, é muito importante conhecer a taxa respiratória e a sensibilidade dos frutos ao $\mathrm{O}_{2}$ e $\mathrm{CO}_{2}$, principalmente para o armazenamento em atmosfera modificada, pois, em função destes dados e da permeabilidade do filme, é possível escolher um material que permita uma atmosfera de armazenamento que diminua o metabolismo dos frutos sem induzir o desenvolvimento de distúrbios fisiológicos decorrentes da respiração anaeróbica.

No metabolismo fermentativo, a produção de etanol envolve a descarboxilação do piruvato a $\mathrm{CO}_{2}$, sem consumo de $\mathrm{O}_{2}$ (FONSECA et al., 2002a). SAQUET \& STREIF (2002) obtiveram resultados que sustentam esta afirmação, nos quais as cultivares de maçã Gala, "Jonagold" e "Fuji", apresentaram redução no consumo de $\mathrm{O}_{2}$, em condições de anaerobiose, sem ocorrer redução na produção de $\mathrm{CO}_{2}$. Assim, esses autores afirmam que o quociente respiratório, relação entre produção de $\mathrm{CO}_{2}$ e consumo de $\mathrm{O}_{2}$, é um parâmetro utilizado para melhor observar a provável ativação das vias fermentativas durante o armazenamento. Segundo KADER (1987), frutos desenvolvendo respiração aeróbica apresentam valores de quociente respiratório entre 0,7 e 1,3, dependendo do substrato que está sendo predominantemente utilizado no processo (FONSECA et al., 2002a). SAQUET \& STREIF (2002) constataram que diversas cultivares de maçãs já iniciaram a respiração anaeróbica em pressões parciais de $\mathrm{O}_{2}$ abaixo de 1,5kPa, atingindo valores de quociente respiratório de 2,5. BRACKMANN et al. (1995) verificaram que $2 \mathrm{kPa}$ de $\mathrm{O}_{2}$ combinados com $5 \mathrm{kPa}$ de $\mathrm{CO}_{2}$ são boas condições para o armazenamento de quivi.
A ocorrência de distúrbios fisiológicos e de sabores e aromas característicos de fermentação não foi observada em quivis armazenados em $2 \mathrm{kPa}$ de $\mathrm{O}_{2}$ combinados com $7 \mathrm{kPa}$ de $\mathrm{CO}_{2}$ (MAZARO et al., 2000). No entanto, neste trabalho, não foram avaliadas pressões parciais de $\mathrm{O}_{2}$ menores que $2 \mathrm{kPa}$ e de $\mathrm{CO}_{2}$ maiores que $7 \mathrm{kPa}$. Segundo IDLER (1997), pressões parciais de $\mathrm{O}_{2}$ inferiores a $1 \mathrm{kPa}$ e de $\mathrm{CO}_{2}$ superiores a $7 \mathrm{kPa}$ induzem o surgimento de degenerescência da polpa em quivi. No entanto, ainda não foram avaliados os efeitos de pressões parciais de $\mathrm{O}_{2}$ menores que $2 \mathrm{kPa}$ e de $\mathrm{CO}_{2}$ maiores que $7 \mathrm{kPa}$ no quivi produzido no Brasil. Assim, é de extrema importância conhecer a combinação de $\mathrm{O}_{2}$ e $\mathrm{CO}_{2}$ que reduza mais acentuadamente a respiração, porém sem induzir o desenvolvimento de degenerescência da polpa e de sabor alcoólico nos frutos.

Dessa forma, o objetivo deste trabalho foi avaliar o efeito de níveis de $\mathrm{O}_{2}$ e $\mathrm{CO}_{2}$ sobre a ocorrência de degenerescência da polpa, de aroma e de sabor alcoólicos no quivi "Bruno" e a relação da ocorrência de degenerescência da polpa e do aroma alcoólico com a taxa respiratória e com o quociente respiratório.

\section{MATERIAL E MÉTODOS}

Os experimentos foram desenvolvidos com quivi (Actinidia chinensis) cultivar "Bruno", no ano de 2004. Os frutos, provenientes de um pomar comercial localizado no município de Farroupilha, após colhidos, foram transportados ao Núcleo de Pesquisa em PósColheita da Universidade Federal de Santa Maria, onde foram descartados os frutos feridos e procedeu-se a homogeneização e a separação das amostras experimentais.

Nos dois experimentos, os tratamentos avaliados foram 0,$5 ; 1,0$ e $1,5 \mathrm{kPa}$ de $\mathrm{O}_{2}$ combinados com 8,12 e $16 \mathrm{kPa}$ de $\mathrm{CO}_{2}$ na temperatura de $0^{\circ} \mathrm{C}$, ambos em um arranjo bifatorial. No experimento 1 , os frutos foram acondicionados em recipientes de vidro, hermeticamente fechados, com capacidade de 5L. No experimento 2, os frutos foram acondicionados em minicâmaras experimentais com capacidade de 60L, hermeticamente fechadas. A unidade experimental foi de 10 frutos e de $5.000 \mathrm{~g}$ de frutos, nos experimentos 1 e 2, respectivamente. Em ambos os experimentos, foram utilizadas três repetições.

As pressões parciais dos gases foram obtidas mediante a diluição do $\mathrm{O}_{2}$ no ambiente de armazenamento com injeção de $\mathrm{N}_{2}$, proveniente de um gerador de nitrogênio que utiliza o princípio Pressure Swing Adsorption - (PSA), com posterior injeção de $\mathrm{CO}_{2}$, proveniente de cilindros de alta pressão, até atingir-se o nível preestabelecido no tratamento. A 
manutenção das pressões parciais desejadas dos gases, nas diferentes condições de armazenamento, que variavam em função da respiração dos frutos, foi realizada duas vezes por dia no experimento 1 e uma vez por dia no experimento 2. Essas avaliações foram feitas através de analisadores eletrônicos de $\mathrm{CO}_{2}$ e $\mathrm{O}_{2}$, marca Agri-datalog, e com posterior correção, até atingiremse os níveis preestabelecidos. $\mathrm{O} \mathrm{O}_{2}$ consumido pela respiração foi reposto por meio da injeção de ar atmosférico nas minicâmaras e o $\mathrm{CO}_{2}$ em excesso foi absorvido por uma solução de hidróxido de potássio ( $40 \%$ p/v), através da qual foi circulado o ar do ambiente de armazenamento.

No experimento 1, para avaliar o efeito dos tratamentos, foram realizadas as determinações da ocorrência de degenerescência da polpa e de aroma alcoólico nos frutos e de sabor alcoólico no suco extraído dos frutos, após 3 meses de armazenamento, a $0^{\circ} \mathrm{C}$, seguidos de dois dias a $20^{\circ} \mathrm{C}$, em atmosfera normal ( $21 \mathrm{kPa}$ de $\mathrm{O}_{2}+0,03 \mathrm{kPa}$ de $\mathrm{CO}_{2}$ ). No experimento 2, foi avaliada a taxa respiratória, em termos de consumo de $\mathrm{O}_{2}$ e produção de $\mathrm{CO}_{2}$, durante o armazenamento nas condições definidas nos tratamentos e calculado o quociente respiratório.

Para avaliar a degenerescência da polpa, os frutos foram cortados transversalmente e realizada a contagem dos que apresentavam regiões internas da polpa com coloração esbranquiçada, sendo os resultados expressos em porcentagem de frutos com incidência de degenerescência de polpa. A presença de aroma alcoólico foi avaliado através de um painel sensorial composto por três pessoas que avaliaram cada fruto, cortado transversalmente, quanto à presença ou não de aroma alcoólico, sendo os resultados expressos em porcentagem de frutos com presença de aroma alcoólico. A presença de sabor alcoólico nos frutos foi determinada através de um painel sensorial composto por seis pessoas, as quais provaram uma amostra de suco extraído dos frutos pertencentes aos tratamentos $0,5 \mathrm{kPa} \mathrm{O}_{2}+8,0 \mathrm{kPa} \mathrm{CO}, 1,0 \mathrm{kPa} \mathrm{O}_{2}+8,0 \mathrm{kPa}$ $\mathrm{CO}_{2}$ e $1,5 \mathrm{kPa} \mathrm{O}_{2}+8,0 \mathrm{kPa} \mathrm{CO}$, indicando a presença ou não de sabor alcoólico.

A taxa respiratória foi determinada pelo consumo de $\mathrm{O}_{2}$ e pela produção de $\mathrm{CO}_{2}$. O gás do espaço livre da minicâmara, utilizada para o acondicionamento das amostras, foi circulado através de analisadores eletrônicos de $\mathrm{O}_{2}$ e $\mathrm{CO}_{2}$, marca Agri-Datalog. Através da concentração de $\mathrm{O}_{2}$ e $\mathrm{CO}_{2}$, do volume do espaço livre, da massa de frutos e do tempo de fechamento, foi calculada a respiração, sendo os valores expressos em $\mathrm{mL}$ de $\mathrm{O}_{2}$ e $\mathrm{CO}_{2} \mathrm{~kg}^{-1} \mathrm{~h}^{-1}$, para o consumo de $\mathrm{O}_{2}$ e a produção de $\mathrm{CO}_{2}$, respectivamente. Também foi calculado o quociente respiratório, através da razão entre a produção de $\mathrm{CO}_{2}$ e o consumo de $\mathrm{O}_{2}$.

A análise de variância seguiu o modelo do delineamento inteiramente casualizado, sendo os dados em porcentagem transformados para $\operatorname{arc}$.sen $\sqrt{x / 100}$, antes de se proceder a análise da variância. As médias foram comparadas pelo teste de Duncan, em nível de $5 \%$ de probabilidade de erro. Também foi realizado o teste de correlação de Pearson entre os parâmetros incidência de degenerescência da polpa, presença de aroma alcoólico (experimento 1), produção de $\mathrm{CO}_{2}$, consumo de $\mathrm{O}_{2}$ e quociente respiratório (experimento 2).

\section{RESULTADOS E DISCUSSÃO}

A ocorrência de degenerescência da polpa e de frutos com aroma alcoólico não foi influenciada pelos níveis de $\mathrm{O}_{2}$ avaliados (Tabela 1). Os tratamentos com $16 \mathrm{kPa}$ de $\mathrm{CO}_{2}$ apresentaram os maiores valores de ocorrência de degenerescência da polpa e de frutos com aroma alcoólico (Tabela 1). Esses resultados demonstram que a ocorrência da degenerescência de polpa está diretamente relacionada com a fermentação da polpa e ambos são desencadeados pelo alto nível

Tabela 1 - Incidência de degenerescência da polpa e de frutos com odor característico de fermentação em quivi “Bruno” em função das pressões parciais de $\mathrm{O}_{2}$ e $\mathrm{CO}_{2}$, após três meses de armazenamento a $0^{\circ} \mathrm{C}$. Santa Maria, 2004.

\begin{tabular}{|c|c|c|c|c|c|c|c|c|}
\hline \multirow{3}{*}{$\mathrm{CO}_{2}(\mathrm{kPa})$} & \multicolumn{4}{|c|}{ Degenerescência da polpa (\%) } & \multicolumn{4}{|c|}{ Odor de fermentação (\%) } \\
\hline & \multicolumn{3}{|c|}{$\mathrm{O}_{2}(\mathrm{kPa})$} & \multirow{2}{*}{ Média } & \multicolumn{3}{|c|}{$\mathrm{O}_{2}(\mathrm{kPa})$} & \multirow{2}{*}{ Média } \\
\hline & 0,5 & 1,0 & 1,5 & & 0,5 & 1,0 & 1,5 & \\
\hline 8,0 & 2,2 & 0,0 & 6,7 & $3,0 \mathrm{c}$ & 2,2 & 0,0 & 8,9 & $3,7 b$ \\
\hline 12,0 & 24,4 & 6,7 & 31,9 & $21,0 \mathrm{~b}$ & 8,9 & 11,1 & 22,5 & $14,2 b$ \\
\hline 16,0 & 79,4 & 63,5 & 80,0 & $74,3 a$ & 63,9 & 77,1 & 86,7 & $75,9 a$ \\
\hline Média & $35,3 \mathrm{~A}$ & $23,4 \mathrm{~A}$ & $39,5 \mathrm{~A}$ & & $25,0 \mathrm{~A}$ & $29,4 \mathrm{~A}$ & $39,4 \mathrm{~A}$ & \\
\hline CV (\%) & & 31,3 & & & & 49,1 & & \\
\hline
\end{tabular}

*Tratamentos com médias não seguidas pela mesma letra, maiúscula na vertical e minúscula na horizontal, diferem pelo teste de Duncan a $5 \%$ de probabilidade de erro. 
de $\mathrm{CO}_{2}$. De acordo com KADER (1987), o $\mathrm{CO}_{2}$ atua reduzindo a velocidade do ciclo dos ácidos tricarboxílicos e, em níveis excessivamente elevados, esta redução pode causar acúmulo de ácido succínico devido à inibição da enzima succinato desidrogenase. LIU et al. (2004), trabalhando com bananas, observaram também uma redução na atividade da enzima isocitrato desidrogenase devido ao alto $\mathrm{CO}_{2}$. Assim, espera-se, devido à redução na velocidade do ciclo dos ácidos tricarboxílicos, uma menor obtenção de energia através da respiração aeróbica. Com isso, os frutos utilizam, como alternativa para a obtenção de energia, a respiração anaeróbica com conseqüente formação de etanol e acetaldeído (PETRACEK et al., 2002) e ocorrência de distúrbios fisiológicos (WATKINS et al., 1997).

Os tratamentos que proporcionaram a menor incidência de degenerescência da polpa e de aroma alcoólico foram aqueles com 0,5, 1,0 e 1,5kPa de $\mathrm{O}_{2}$ combinados com $8 \mathrm{kPa}$ de $\mathrm{CO}_{2}$ (Tabela 1 ). No entanto, de acordo com a análise sensorial, o suco extraído dos frutos armazenados em $1,0 \mathrm{kPa} \mathrm{O}_{2}+8,0 \mathrm{kPa} \mathrm{CO}_{2}$ apresentou melhor sabor e alguns avaliadores consideraram que o suco extraído dos frutos armazenados em $0,5 \mathrm{kPa} \mathrm{O}_{2}+8,0 \mathrm{kPa} \mathrm{CO}_{2}$ apresentou sabor alcoólico (dados não apresentados). Com relação ao efeito do $\mathrm{CO}_{2}$, os resultados do presente trabalho discordam daqueles obtidos por IDLER (1997), que afirma que pressões parciais de $\mathrm{CO}_{2}$ acima de $7 \mathrm{kPa}$ induzem a ocorrência de degenerescência da polpa. No entanto, com relação ao efeito do $\mathrm{O}_{2}$, os resultados deste trabalho estão de acordo com os de IDLER (1997), pois este autor observou que pressões parciais de $\mathrm{O}_{2}$ inferiores a $1 \mathrm{kPa}$ induzem o surgimento de danos em quivi.

Observou-se interação entre os níveis de $\mathrm{O}_{2}$ e $\mathrm{CO}_{2}$ para as variáveis dependentes taxa de consumo de $\mathrm{O}_{2}$ e quociente respiratório (Tabela 2). A taxa de produção de $\mathrm{CO}_{2}$ não foi influenciada pelos níveis de $\mathrm{O}_{2}$ (Tabela 2). Quanto ao nível de $\mathrm{CO}_{2}$, a taxa de produção de $\mathrm{CO}_{2}$ foi menor nos tratamentos com $16 \mathrm{kPa}$, seguidos pelos tratamentos com $12 \mathrm{kPa}$ de $\mathrm{CO}_{2}$ (Tabela 2). LIU et al. (2004) também observaram redução na produção de $\mathrm{CO}_{2}$, em bananas, pelo alto nível de $\mathrm{CO}_{2}$. O consumo de $\mathrm{O}_{2}$ foi menor no tratamento com $0,5 \mathrm{kPa}$ de $\mathrm{O}_{2}$ combinado com $16 \mathrm{kPa}$ de $\mathrm{CO}_{2}$ seguido pelos tratamentos com 1,0 e $1,5 \mathrm{kPa}$ de $\mathrm{O}_{2}$ combinados com $16 \mathrm{kPa}$ de $\mathrm{CO}_{2}$ (Tabela 2). Este resultado evidencia um efeito combinado entre o baixo $\mathrm{O}_{2}$ e o alto $\mathrm{CO}_{2}$ sobre o consumo de $\mathrm{O}_{2}$. FONSECA et al. (2002b) também verificaram menor atividade respiratória com a redução do nível de $\mathrm{O}_{2}$ e o aumento do $\mathrm{CO}_{2}$. De acordo com KADER (1986), o baixo $\mathrm{O}_{2}$ reduz a taxa respiratória devido à redução da atividade das enzimas citocromo oxidase, polifenoloxidases, ácido ascórbico oxidase e ácido glicólico oxidase. MATHOOKO (1996) e FONSECA et al. (2002a) afirmam que o $\mathrm{CO}_{2}$ é um forte inibidor do processo respiratório em alguns frutos, sendo que seu efeito pode ser direto, atuando sobre a rota glicolítica, o ciclo dos ácidos tricarboxílicos e o sistema transportador de elétrons, ou então indireto, atuando como antagonista à ação do etileno sobre algumas enzimas envolvidas no processo respiratório.

$\mathrm{O}$ quociente respiratório foi maior no tratamento com $0,5 \mathrm{kPa}$ de $\mathrm{O}_{2}$ combinado com $16 \mathrm{kPa}$ de $\mathrm{CO}_{2}$ (Tabela 2). PETRACEK et al. (2002), trabalhando com cerejas, também observaram maior quociente respiratório quando os frutos foram armazenados em baixo $\mathrm{O}_{2}$. De acordo com KADER (1987), frutos que apresentam quociente respiratório acima de 1,3 podem estar desenvolvendo respiração anaeróbica. Este resultado está de acordo com os obtidos no presente trabalho, pois os tratamentos que apresentaram

Tabela 2 - Taxa respiratória, em termos de produção de $\mathrm{CO}_{2}$ e consumo de $\mathrm{O}_{2}$, e quociente respiratório de quivi "Bruno” em função das pressões parciais de $\mathrm{O}_{2}$ e $\mathrm{CO}_{2}$, após três meses de armazenamento a $0^{\circ} \mathrm{C}$. Santa Maria, 2004.

\begin{tabular}{|c|c|c|c|c|c|c|c|c|c|c|}
\hline \multirow{3}{*}{$\mathrm{CO}_{2}(\mathrm{kPa})$} & \multicolumn{4}{|c|}{$\begin{array}{c}\text { Produção de } \mathrm{CO}_{2} \\
\left(\mathrm{~mL} \text { de } \mathrm{CO}_{2} \mathrm{~kg}^{-1} \mathrm{~h}^{-1}\right)\end{array}$} & \multicolumn{3}{|c|}{$\begin{array}{l}\text { Consumo de } \mathrm{O}_{2} \\
\left(\mathrm{~mL} \text { de } \mathrm{O}_{2} \mathrm{~kg}^{-1} \mathrm{~h}^{-1}\right)\end{array}$} & \multicolumn{3}{|c|}{ Quociente respiratório $\left(\mathrm{CO}_{2} / \mathrm{O}_{2}\right)$} \\
\hline & \multicolumn{3}{|c|}{$\mathrm{O}_{2}(\mathrm{kPa})$} & \multirow{2}{*}{ Média } & \multicolumn{3}{|c|}{$\mathrm{O}_{2}(\mathrm{kPa})$} & \multicolumn{3}{|c|}{$\mathrm{O}_{2}(\mathrm{kPa})$} \\
\hline & 0,5 & 1,0 & 1,5 & & 0,5 & 1,0 & 1,5 & 0,5 & 1,0 & 1,5 \\
\hline 8,0 & 1,18 & 1,19 & 1,23 & $1,20 \mathrm{a}$ & 0,99Ba & 1,31Aa & 1,32Aa & $1,20 \mathrm{Ab}$ & $0,90 \mathrm{Bc}$ & $0,93 \mathrm{Bb}$ \\
\hline 12,0 & 0,99 & 0,96 & 1,02 & $0,98 \mathrm{~b}$ & $0,78 \mathrm{Bb}$ & $0,88 \mathrm{Ab}$ & $0,78 \mathrm{Bb}$ & $1,27 \mathrm{Ab}$ & $1,07 \mathrm{Bb}$ & $1,32 \mathrm{Aa}$ \\
\hline 16,0 & 0,92 & 0,94 & 0,92 & $0,93 \mathrm{c}$ & $0,58 \mathrm{Bc}$ & 0,69Ac & $0,68 \mathrm{Ac}$ & 1,59Аа & $1,38 \mathrm{Ba}$ & $1,36 \mathrm{Ba}$ \\
\hline Média & $1,03 \mathrm{~A}$ & $1,03 \mathrm{~A}$ & $1,06 \mathrm{~A}$ & & - & - & - & - & - & - \\
\hline CV (\%) & & 4,55 & & & & 5,29 & & & 4,28 & \\
\hline
\end{tabular}

*Tratamentos com médias não seguidas pela mesma letra, maiúscula na vertical e minúscula na horizontal, diferem pelo teste de Duncan a $5 \%$ de probabilidade de erro 
quociente respiratório acima de 1,3 foram aqueles que apresentaram a maior incidência de frutos com degenerescência da polpa e com aroma alcoólico (Tabelas 1 e 2). PETRACEK et al. (2002) observaram que os tratamentos com maior quociente respiratório apresentaram maior acúmulo de produtos do metabolismo fermentativo, os quais conferem sabor e aroma alcoólicos aos frutos.

Com relação à análise de correlação de Pearson, verificou-se uma correlação positiva entre o quociente respiratório e a ocorrência de degenerescência da polpa $\left(\mathrm{R}^{2}=0,8100\right)$ e a presença de odor de fermentação $\left(\mathrm{R}^{2}=0,6574\right)$. Observou-se também uma correlação negativa entre a ocorrência de degenerescência da polpa e o consumo de $\mathrm{O}_{2}\left(\mathrm{R}^{2}=-0,7823\right)$ e a produção de $\mathrm{CO}_{2}\left(\mathrm{R}^{2}=-0,7197\right)$ e entre a presença de odor de fermentação com o consumo de $\mathrm{O}_{2}\left(\mathrm{R}^{2}=-0,6584\right)$ e a produção de $\mathrm{CO}_{2}\left(\mathrm{R}^{2}=-0,6383\right)$. Estes resultados demonstram que o quociente respiratório e a atividade respiratória podem ser indicadores de condições de armazenamento indutoras de distúrbios fisiológicos decorrentes de respiração anaeróbica em quivi. Este resultado concorda com a afirmação de SAQUET \& STREIF (2002), que afirmam que o quociente respiratório é um parâmetro utilizado para melhor monitorar a indução das vias fermentativas durante o armazenamento de maçãs em atmosfera controlada. PETRACEK et al. (2002) afirmam que, em cerejas, a indução da respiração anaeróbica está correlacionada com o quociente respiratório. Observa-se também que o maior quociente respiratório, nos tratamentos que apresentaram elevada incidência de degenerescência da polpa, ocorreu por uma grande produção de $\mathrm{CO}_{2}$, sem haver consumo proporcional de $\mathrm{O}_{2}$ (Tabela 2). SAQUET \& STREIF (2002) também verificaram este comportamento em maçãs armazenadas em condições de anaerobiose. Segundo TAIZ \& ZEIGER (2004), em condições de anaerobiose, as células vegetais, para fornecer energia para a manutenção celular sem o uso de oxigênio, realizam a fermentação alcoólica. Neste processo, o piruvato pode ser utilizado pelas enzimas piruvato descarboxilase e a álcool desidrogenase, liberando $\mathrm{CO}_{2}$ e etanol (PETRACEK et al., 2002; TAIZ \& ZEIGER, 2004).

\section{CONCLUSÃO}

A melhor condição de atmosfera testada para o armazenamento do quivi cultivar "Bruno" a $0^{\circ} \mathrm{C}$ é a de $1 \mathrm{kPa}$ de $\mathrm{O}_{2}+8 \mathrm{kPa}$ de $\mathrm{CO}_{2}$. A incidência de degenerescência e de aroma alcoólico na polpa do quivi "Bruno" possui correlação positiva com o quociente respiratório e negativa com a respiração dos frutos.
$\mathrm{O} \mathrm{O}_{2}$ abaixo de $1 \mathrm{kPa}$ induz a formação de sabor alcoólico nos frutos e o $\mathrm{CO}_{2}$ acima de $8 \mathrm{kPa}$ causa uma alta ocorrência de degenerescência da polpa.

\section{REFERÊNCIAS}

BRACKMANN, A. et al. Armazenamento refrigerado de kiwi em atmosfera normal e controlada. Revista Brasileira de Agrociência, Pelotas, v.1, n.2, p.107-111, 1995.

FONSECA, S.C. et al. Modelling respiration rate of fresh fruits and vegetables for modified atmosphere packages: a review. Journal of Food Engeneering, Amsterdam, n.52, p.99-119, 2002a

FONSECA, S.C. et al. Modelling respiration rate of shredded Galega kale for development of modified atmosphere packaging. Journal of Food Engeneering, Amsterdam, n.54, p.299307, 2002b.

IDLER, L. Overview of controlled atmosphere transportation in containers. In: INTERNATIONAL CONTROLLED ATMOSPHERE RESEARCH CONFERENCE, 7., 1997, Davis, California. Proceedings... Davis:Postharvest Horticulture Series, 1997. V.1, n.15, p.2-10.

KADER, A.A. Biochemical and physiological basis of effects of controlled and modified atmospheres on fruits and vegetables. Food Technology, Alexandria, v.40, n.5, p.99-104, 1986.

KADER, A.A. Respiration and gas exchange of vegetables. In: WEICHMANN, J. Postharvest physiology of vegetables, New York: Marcel Dekker, 1987. p.25-43.

KE, D. et al. Regulation of fermentative metabolism in fruits and vegetables by controlled atmospheres. In: INTERNATIONAL CONTROLLED ATMOSPHERE RESEARCH CONFERENCE, 6., 1993, Ithaca, New York. Proceedings... Ithaca, New York: Postharvest Horticulture Series, 1993. V.1, p.63-67.

LIU, S. et al. Effects of $\mathrm{CO}_{2}$ on respiratory metabolism in ripening banana fruit. Postharvest Biology and Technology, Amsterdam, n.33, p.27-34, 2004

MAHAJAN, P.V.; GOSWANI, T.K. Enzyme kinetics based modeling of respiration rate of apple. Journal Agricultural Engeneering Research, Amsterdam, v.79, n.4, p.399-406, 2001.

MATHOOKO, F.M. Regulation of respiratory metabolism in fruits and vegetables by carbon dioxide. Postharvest Biology and Technology, Amsterdam, v.9, p.247-264, 1996.

MAZARO, S.M. et al. Qualidade de kiwi armazenado em duas temperatures sob atmosfera controlada e com eliminação de etileno. Ciência Rural, Santa Maria, v.30, n.6, p.947-952, 2000.

PETRACEK, P.D. et al. Modified atmosphere packaging of sweet cherry (Prunus avium L., cv. 'Sams') fruit: metabolic responses to oxygen, carbon dioxide, and temperature. Postharvest Biology and Technology, Amsterdam, n.24, p.259-270, 2002. 
SAQUET, A.A.; STREIF, J. Respiração e produção de etileno de maçãs armazenadas em diversas concentrações de oxigênio. Revista Brasileira de Agrociência, Pelotas, v.8, n.1, p.7175, 2002.

SAQUET, A.A.; STREIF, J. Untersuchungen zur Atmung und zur Ethylenbildung einiger neuer Apfelsorten. Erwerbsobstbau, Berlim, v.42, p.109-112, 2000.

SAQUET, A.A. et al. Changes in ATP, ADP and pyridine nucleotide levels related to the incidence of physiological disorders in 'Conference' pears and 'Jonagold' apples during controlled atmosphere storage. Journal of Horticultural Science \& Biotechnology, Ashford, v.75, p.243-249, 2000.
SHIINA, T. et al. Effects of temperature and gas concentration on the respiration of fruits and vegetables. In: INTERNATIONAL CONTROLLED ATMOSPHERE RESEARCH CONFERENCE, 7., 1997, Davis, California. Proceedings... Davis, California: Postharvest Horticulture Series, 1997. V.15, n.1, p.71-76.

TAIZ, L.; ZEIGER, E. Respiração e metabolismo de lipídios. In:__ Fisiologia vegetal. Porto Alegre: Artmed, 2004. Cap.11, p.251-284.

WATKINS, C.B. et al. A comparison of two carbon dioxiderelated injuries of apple fruit. In: INTERNATIONAL CONTROLLED ATMOSPHERE RESEARCH CONFERENCE, 7., 1997, Davis, California. Proceedings... Davis, California: Postharvest Horticulture Series, 1997. V.2, p.119-124. 1997. 